\title{
An optimization of microwave-assisted grafting of poly $(N$-isopropylacrylamide) (PNIPAM) onto nylon-6 porous film for thermo-responsive gating membrane
}

\author{
Putita KATESRIPONGSA* and Tatiya TRONGSATITKUL* \\ *School of Polymer Engineering, Suranaree University of Technology \\ 111 University, Muang, Nakhon Ratchasima 30000, Thailand \\ E-mail: tatiya@sut.ac.th
}

Received: 11 January 2019; Revised: 14 March 2019; Accepted: 15 April 2019

\begin{abstract}
This work focused on a fabrication of smart membranes using a novel grafting method comprising of plasma peroxide technique together with microwave-assisted polymerization. PNIPAm, thermo-responsive polymer, both with and without crosslinker, were grafted onto porous Nylon-6 membranes. Key parameters for grafting including the microwave irradiation time, output power, and monomer concentration have been investigated. Characterizations using scanning electron microscope (SEM) and Fourier-transform infrared spectroscopy (FTIR) revealed that the PNIPAm have been successfully grafted onto the surface both on the top surface of the membrane and the surface inside the pores. Irradiation time of $10 \mathrm{~min}$ under $800 \mathrm{w}$ were found to be an optimum condition which gave the highest yield of $10 \mathrm{wt} \%$. For both the linear and crosslinked PNIPAm-grafted membranes, the highest grafting yield was obtained from using $3 \mathrm{wt} \%$ of NIPAm monomer solution. Because of this fast fabrication time and environmentally friendly method, new smart gating membranes can be produced which will help providing an ever-better performances transport control of gas and liquid for various fields including medical and packaging applications.
\end{abstract}

Keywords : Porous nylon-6 (PA6) membrane, Poly( $N$-isopropylacrylamide), Thermoresponsive polymer, Microwave-assisted polymerization, Plasma treatment, Smart gating

\section{Introduction}

Smart polymers or stimuli-responsive polymers are polymers that respond with a considerable change in their properties to different stimuli or changes in the environment. This type of polymer may be classified according to the stimuli it responds to including temperature, $\mathrm{pH}$, photo, or enzyme. Poly $(N$-isopropylacrylamide $)$ or PNIPAm is a smart polymer which has been extensively studied. This is because PNIPAm is a thermo-responsive polymer that possesses a well-defined lower critical solution temperature or LCST at around $32^{\circ} \mathrm{C}$ (Xie et al., 2007). This temperature is useful as it is close to physiological temperature which can also be adjusted or tailored by copolymerization with another monomer (Trongsatitkul and Budhlall, 2011). This is the main reason that most researchers focus to exploit PNIPAm in medical field such as controlled release (Chung - Yang et al., 2009), tissue engineering (Stile and Healy, 2001), and immobilization of enzymes (Liu et al., 2012).

Another use of PNIPAm that has been attracting wide interests over the last decade is a smart gating application. In this case, permeation properties can be controlled or adjusted by the gates according to an environmental temperature. To be specific, PNIPAm chains are attached or graft onto a porous membrane's surface. Collapses of PNIPAm chains as temperature increases above the LCST are exploited as an open/close mechanism of the pores or gates on the membrane. Several applications could find these smart gating membranes advantageous including controlled drug delivery, bioseparation, chemical separation, water treatment, chemical sensors, and tissue engineering.

Different applications require different gating performance. It has been shown that linear chain PNIPAm generally 
gives a sharp response to temperature change (Schild, 1992). However, a three-dimensional crosslink network of PNIPAm may be better in term of open/close gating performance (Chen et al., 2009).

Beside the form of grafted PNIPAm on the membrane surface, type of substrate used also affects gating performance. Many stimuli-responsive gating membranes have been investigated such as PNIPAm-g-Nylon-6 (Yang et al., 2006), PNIPAm-g-PP (Choi et al., 2000), PNIPAm-g-PVDF (Yang et al., 2006), PNIPAm-g-PC (Ohashi et al., 2016), and PNIPAm-g-HDPE (Yamaguchi et al., 1999) membranes. Comparative study of smart gating membranes with different substrates on gating performance were carried out by Yang et al.(2006). By measuring the water flux over the temperature range of $25-40^{\circ} \mathrm{C}$, it was found that the water flux through the hydrophilic Nylon-6 substrate was higher than that of through the hydrophobic HDPE substrate at the same temperature (Yang et al., 2006) which was due to the differences in the substrate properties, specifically its hydrophilicity and microstructure.

Several grafting techniques have been developed to graft PNIPAm onto a membrane such as plasma induced graft polymerization (PIGP) (CHU Liangyin, 2003), UV radiation induced grafting, plasma graft copolymerization, atom transfer radical polymerization (ATRP) (Xu et al., 2007), and plasma-graft pore-filling polymerization (Xie et al., 2005). Microwave irradiation can also be used to assist the grafting process. There are several unique advantages in using microwave irradiation for polymerization including low energy consumption and thermal homogeneity (Oliver, 2004). Earlier work reported a successful use of microwave-assisted heating for synthesize PNIPAm hydrogels which shortened the reaction time of $6 \mathrm{~h}$ down to $1 \mathrm{~h}$ (Murray et al., 1994). However, to the best of the authors' knowledge, the microwaveassisted grafting technique of PNIPAm onto Nylon porous membrane has not been investigated.

In this study, plasma peroxide method together with microwave-assisted polymerization were used to graft PNIPAm, onto a porous Nylon-6 membrane. This was because Nylon membrane was hydrophilic which featured porous honeycomb microstructure below the skin layer that can be effectively grafted with PNIPAm and exhibited a high gating performance. The focus of this work was to optimize the key parameters involving with microwave-assisted grafting. These key parameters included the effect of the microwave output power and irradiation time as well as concentration of the monomer solution on grafting yield. The presence of crosslinker in the monomer solution was also investigated for its effects on the grafting yield and, to be reported later (elsewhere), gating performance. Fourier transform infrared spectrometry (FTIR) and scanning electron microscopy (SEM) were used to verify the success of the grafting using the novel technique.

\section{Experimental methods}

\subsection{Materials}

The Nylon membranes used in this study were from Whatman Co. They possessed an average pore size of $0.2 \mu \mathrm{m}$ and thickness of 150-187 $\mu \mathrm{m}$. $N$-isopropylacrylamide (NIPAm) (98\%) and $N, N^{\prime}$-methylenebisacrylamide (BIS), used as the main monomer and a crosslinker, respectively and were purchased from TCI Co. The monomer and crosslinker were used without further purification. Deionized water was used in all experiment throughout the study.

\subsection{Grafting of linear and crosslinked PNIPAm onto porous nylon 6 substrates by plasma peroxide method together with microwave-assisted polymerization}

The mechanism for grafting PNIPAm onto nylon porous membrane surfaces is schematically depicted as shown in Fig. 1.

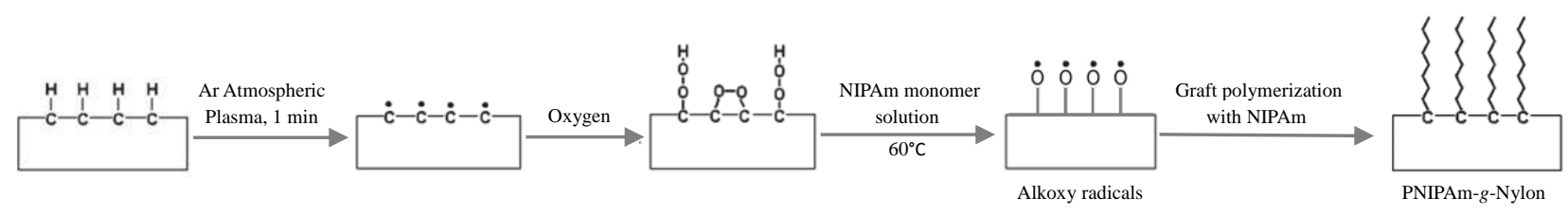

Fig. 1 Grafting of NIPAm onto Nylon-6 porous membrane's surface via plasma peroxide method followed by microwaveassisted polymerization. 
Firstly, as-received Nylon 6 membranes were treated with argon plasma at $30 \mathrm{~W}$ for $60 \mathrm{~s}$ to activate the surface of the polymer membranes and to form free radicals. These activated membranes were then exposed to oxygen in the atmospheric air for 2 days so that the free radicals reacted with oxygen and formed hydrogen peroxide on the membrane's surface. All the parameters in the first step were kept constant.

Secondly, NIPAm monomers were grafted onto the membrane's surface using microwave-assisted polymerization. In this step, the treated membranes were submerged in NIPAm monomer solution before being irradiated with microwave to induce grafting polymerization by heating. Typically, as the peroxides being the assumed initiator, the applied grafting temperature is normally higher than $60^{\circ} \mathrm{C}$ to assure the decomposition of peroxides to produce alkoxy radicals in aqueous solution, thereby initiating the polymerization (Xueqin et al., 2014). Effects of the main parameters in the second step i.e. microwave irradiation output power and time, the presence of crosslinker, and the concentration of the monomer concentration on the grafting effectiveness or grafting yield were examined. Optimum condition for grafting of PNIPAm onto the porous Nylon membrane was expected to obtain from this investigation. The optimization of microwave assisted polymerization step was carried out as followed.

1. To study effect of microwave irradiation output power and time, the monomer solution comprised of $10 \mathrm{wt} \%$ NIPAm in water was used. A treated membrane was fully submerged into the monomer solution in a Petri dish. Irradiation of microwave power and time were varied from 100 to $800 \mathrm{w}$ and 5 to $15 \mathrm{~min}$, respectively. After completion of microwave irradiation step. The grafted membrane was repeatedly rinsed with DI water before mildly sonicated in a temperature-controlled bath $\left(30^{\circ} \mathrm{C}\right)$ for $24 \mathrm{~h}$ to ensure the removal of any residual unreacted chemicals. Finally, the grafted membrane was dried in an oven at $50^{\circ} \mathrm{C}$ for $12 \mathrm{~h}$ before being tested or characterized.

2. The study of the effects of the presence of BIS crosslinker and the monomer solution concentration were performed by using the optimum microwave irradiation condition obtained from the prior step. The monomer solutions, with and without crosslinker, with a constant solid content were prepared. In the case of the solution with the presence of crosslinker, the constant weight ratio of 800:50 (NIPAm:BIS) was used. The monomer solution concentrations varied from 2 to $10 \mathrm{wt} \%$ were prepared. The grafting of the treated membranes with the different monomer solutions were carried out as described above. Different conditions used for optimization in this study were summarized in Table 1.

Table 1 Conditions for the PNIPAm- $g$-Nylon membranes.

\begin{tabular}{|c|c|c|c|}
\hline Condition no. & $\begin{array}{c}\text { NIPAm } \\
(\mathrm{wt} \%)\end{array}$ & $\begin{array}{c}\begin{array}{c}\text { Output power } \\
(\mathrm{w})\end{array} \\
\end{array}$ & $\begin{array}{l}\text { Irradiation } \\
\text { time (min) }\end{array}$ \\
\hline 1 & \multirow{9}{*}{10} & \multirow{3}{*}{100} & 5 \\
\hline 2 & & & 10 \\
\hline 3 & & & 15 \\
\hline 4 & & \multirow{3}{*}{450} & 5 \\
\hline 5 & & & 10 \\
\hline 6 & & & 15 \\
\hline 7 & & \multirow{3}{*}{800} & 5 \\
\hline 8 & & & 10 \\
\hline 9 & & & 15 \\
\hline 10 & 7 & \multirow{4}{*}{\multicolumn{2}{|c|}{ Optimum condition }} \\
\hline 11 & 5 & & \\
\hline 12 & 3 & & \\
\hline 13 & 2 & & \\
\hline
\end{tabular}

The grafted membranes obtained from the different compositions were characterized for their grafting effectiveness via FTIR, SEM, and weighing method for the proof of reaction, change in structure, and grafting yield, respectively. The grafting yield based on membrane weight was calculated according to the following Eq. (1).

$$
Y=\frac{W_{g}-W_{0}}{W_{0}} \times 100 \%
$$


where $\mathrm{Y}$ is the grafting yield of PNIPAm on the membrane substrate, $\mathrm{W}_{\mathrm{g}}$ and $\mathrm{W}_{0}$ are the mass of the membrane after and before grafting, respectively (Yang et al., 2006).

\subsection{Morphological analysis}

Scanning electron microscopy (SEM) (JSM-6010LV, JEOL, Japan) was employed to observe the microstructures of the membranes, both outer surface and cross-sectional area. The cross-sectional area of the membranes were prepared by cryo-fractured in liquid nitrogen. All the surfaces were sputter-coated with gold for $60 \mathrm{~s}$ before observation with SEM at an accelerating voltage of $10 \mathrm{kV}$.

\subsection{Chemical composition analysis}

Fourier Transform Infrared Spectrometer (FT-IR) (Tensor 27, Bruker, Billerica, MA, USA) was used to confirm the grafting of PNIPAm polymer onto the Nylon 6 porous membranes. The attenuated total reflection (ATR) mode was used for all the samples. The spectra were collected at $4 \mathrm{~cm}^{-1}$ resolution with an FT-IR microscopic spectrometer over 64 scans.

\section{Results and discussion}

\subsection{Confirmation of grafted PNIPAm on nylon membranes}

The effects of microwave irradiation time and output power on the success of grafting of PNIPAm onto Nylon-6 porous membranes were first investigated. The results shown in Fig. 2 illustrated the effect of the output power and irradiation time on grafting yield at a constant monomer content of $10 \mathrm{wt} \%$. It can be seen that PNIPAm- $g$-Nylon membranes with a grafting yield of 2.85-11.2 \% and 11.7-18.3\% were obtained for linear and crosslinked PNIPAmgrafted membranes, respectively. For both the linear and crosslinked PNIPAM- $g$-Nylon porous membranes, the condition for microwave irradiation that gave the highest yield were found to be $800 \mathrm{~W}$ and $10 \mathrm{~min}$. Note that, with the presence of crosslinker in the monomer solution, grafting yield was about 4 times greater than that of without one.

Different yields obtained from the use of different microwave irradiation time and power during microwave-assisted polymerization were due to the different temperatures were induced. In this Novel grafting method, temperature of $60^{\circ} \mathrm{C}$ or above is required to produce alkoxy radicals from peroxides. Figure 3 shows the temperatures measured on the surface of Nylon using IR thermosensor immediately after microwave irradiation. The temperatures of the membranes were varied from 44 to $92^{\circ} \mathrm{C}$. It should be noted that due to the time lag of the temperature measurement, the actual temperature during the irradiation may be significantly higher than these value. In general, however, it can be seen that higher temperature and longer time gave higher yield. The results infer that the grafting yield is time and temperature dependent. However, there seemed to be the limiting or critical temperature at $87^{\circ} \mathrm{C}$ that gave the maximum yields. Further increased the grafting temperature resulted in declining of the grafting yield. This result is similar to that of reported by Xueqin et al.(2014). In general, a decrease in grafting yield at elevated temperature is known and accepted as a consequence of an acceleration of the termination reaction. The increased temperature causes faster rates of peroxide decomposition, free radical consumption, and termination.

Regarding of the grafting temperature, it should also be noted that all conditions used here induced temperature significantly higher than the LCST of NIPAm $\left(32^{\circ} \mathrm{C}\right)$. Thus, during the grafting process, PNIPAm chains were assumed to aggregate in all conditions. The aggregation of the polymer chains may partially be responsible for low grafting yield, particularly in the case when a high monomer concentration solution was used. However, the yield obtained in this study at low concentration such as at $3 \mathrm{wt} \%$ was comparable to that of reported by others (Yang et al., 2006). This might plausibly be due to that the pores were pre-filled with enough amount of monomer within. The aggregation of the polymer, that could have prevented the permeation of monomer molecules from solution outside the pore, therefore show no effect on the grafting yield. This was only the case for monomer solution with low concentration, but not for the higher concentration one. The effect of monomer concentration is discussed in this report in the later section.

The success of grafting using the novel technique was confirmed by ATR-FTIR technique shown in Fig. 4. 
Characteristic peak of isopropyl group at $1385 \mathrm{~cm}^{-1}$ appeared newly in the spectra of the PNIPAm-grafted membranes which prepared using microwave irradiation at $450 \mathrm{w}$ for $15 \mathrm{~min}$ and $800 \mathrm{w}$ for 10 and $15 \mathrm{~min}$. From FTIR spectra, FTIR index ( $\left.\mathrm{T}_{\text {neat }} / \mathrm{T}_{\text {grafted }}\right)$ was calculated and summarized in Table 2. The maximum index value was observed in the grafted sample prepared under $800 \mathrm{w}$ for $10 \mathrm{~min}$ with the presence of crosslinker. The result was well agreed with the calculated grafting yield. However, this was not the case for other conditions. This might be due to an inhomogeneous distribution of grafted PNIPAm on the substrate.

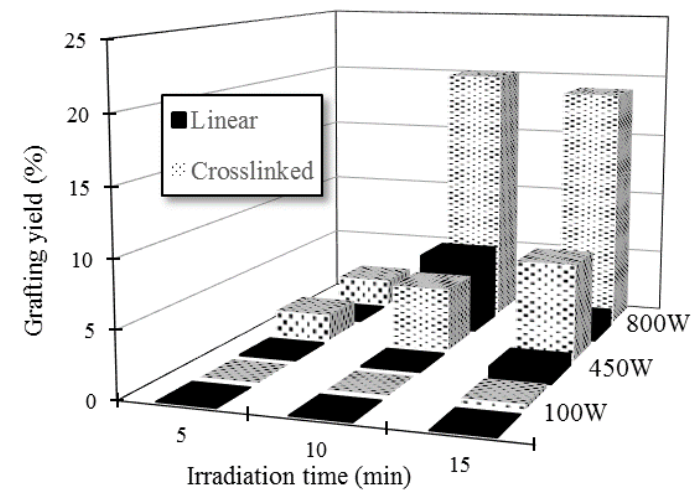

Fig. 2 Grafting yield of PNIPAm- $g$-Nylon membrane using different microwave irradiation time and output power with a constant $10 \mathrm{wt} \%$ NIPAm solution.

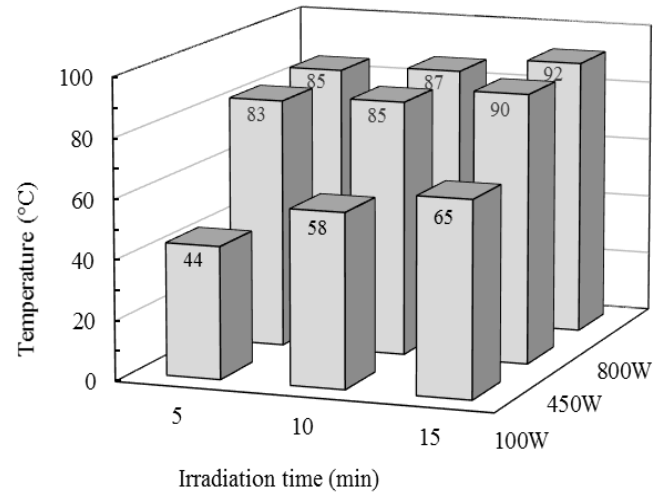

Fig. 3 Temperature of Nylon membrane at different microwave irradiation time and output power. (measure by infrared thermometer)

(a)

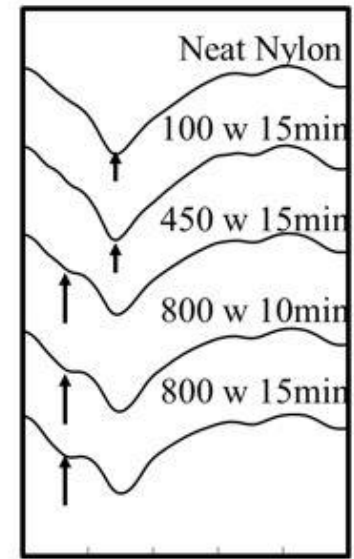

140013801360134013201300 Wavenumber $\left(\mathrm{cm}^{-1}\right)$ (b)

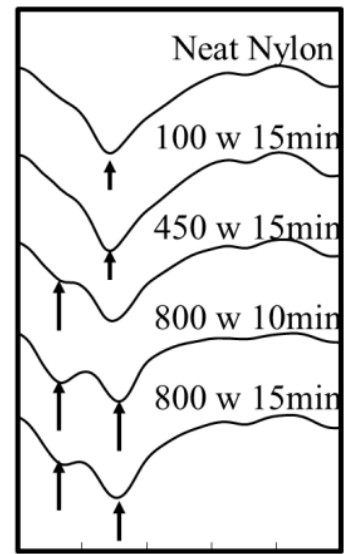

140013801360134013201300 Wavenumber $\left(\mathrm{cm}^{-1}\right)$

Fig. 4 FTIR/ATR spectra of membranes: (a) linear, and (b) crosslinked PNIPAm- $g$-Nylon membrane. 
Table 2 FTIR index of membranes for grafting optimization.

\begin{tabular}{|c|c|c|c|}
\hline & \multicolumn{2}{|c|}{ Condition } & Index \\
\hline \multirow{7}{*}{ Linear } & $\begin{array}{c}\text { Output power } \\
\text { (w) }\end{array}$ & Time (min) & $1385 \mathrm{~cm}^{-1}$ \\
\hline & \multirow{3}{*}{450} & 5 & 1.005 \\
\hline & & 10 & 1.010 \\
\hline & & 15 & 1.019 \\
\hline & \multirow{3}{*}{800} & 5 & 1.008 \\
\hline & & 10 & 1.007 \\
\hline & & 15 & 1.019 \\
\hline \multirow{6}{*}{ Crosslinked } & \multirow{3}{*}{450} & 5 & 0.994 \\
\hline & & 10 & 1.041 \\
\hline & & 15 & 1.085 \\
\hline & \multirow{3}{*}{800} & 5 & 1.000 \\
\hline & & 10 & 1.215 \\
\hline & & 15 & 1.203 \\
\hline
\end{tabular}

The relationship between the grafting yield and NIPAm concentration is shown in Fig. 5. For both the linear and crosslinked PNIPAm-grafted membranes, the highest grafting yield was obtained from using 3 wt $\%$ of NIPAm monomer solution. Further increased PNIPAm concentration above $3 \mathrm{wt} \%$ gave lower yield suggesting that $3 \mathrm{wt} \%$ was a critical concentration to obtain highest degree of grafting. The plausible explanation was to be explained in the following section that reported about morphology of the grafted membrane.

It was again observed that the presence of crosslinker gave higher grafting yield than that of without one. Characteristic peak of isopropyl group at $1385 \mathrm{~cm}^{-1}$ appeared in the FTIR spectrum for grafted samples both linear (without crosslinker) and crosslinked PNIPAm-grafted membranes as shown in Fig. 6.

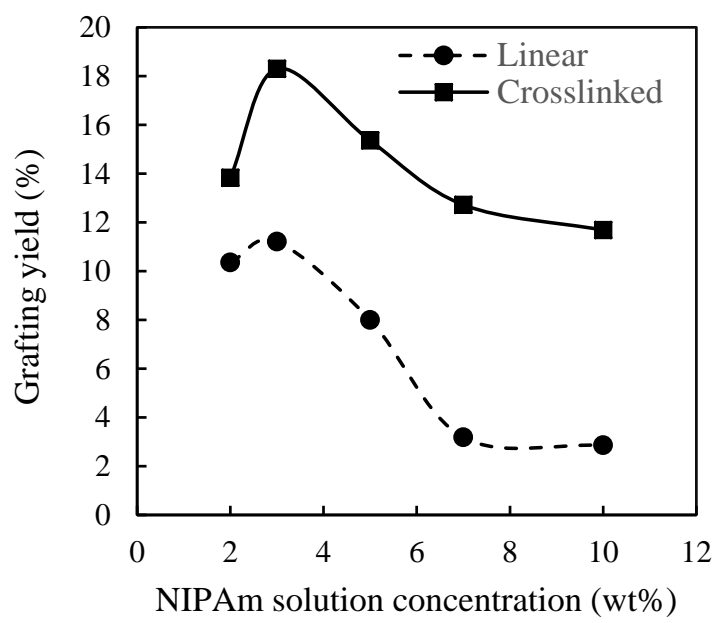

Fig. 5 Relationship between the NIPAm monomer solution concentration and the grafting yield onto Nylon-6 porous membrane. 
(a)

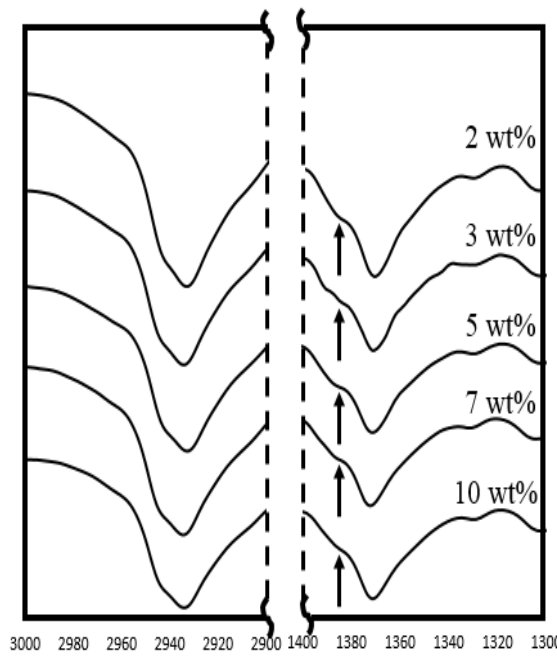

(b)

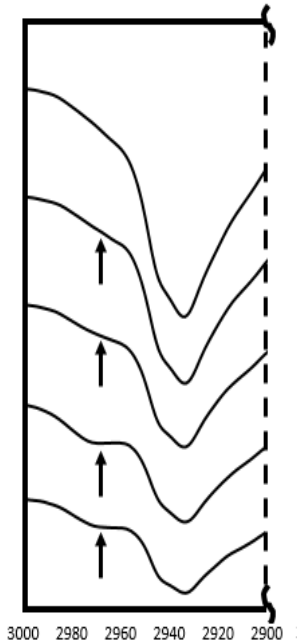

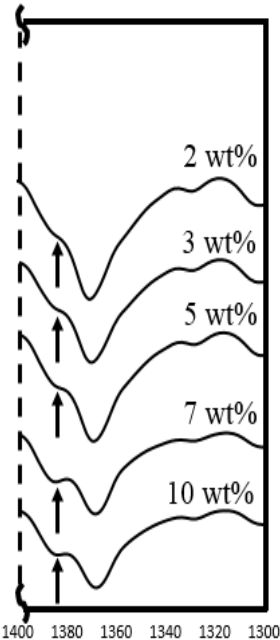

Fig. 6 FTIR/ATR spectra of Nylon-6 porous membranes grafted with: (a) linear, and (b) crosslinked PNIPAm using different monomer concentrations.

\subsection{Morphological analysis of PNIPAm-grafted membranes}

Morphologies of the grafted membranes were analyzed using SEM. SEM micrographs shown in Fig. 7 were a cryofreeze fractured cross-section surface of the grafted membranes. It was described earlier that initially, the membrane was only immersed in the monomer solution before being irradiated with microwave, which result in a dense PNIPAm grafted mostly onto top surface of membrane. Attempting to improve grafting homogeneity, a pressure-assisted permeation of monomer solution was carried out. Adaptation of coffee maker "Aeropress" was employed to help forcing monomer solution to penetrate into the pores of the membrane. An improvement of grafting was observed as better uniformity of grafting of PNIPAm onto the porous Nylon was found and PNIPAm grafted onto the surface of deeper pores.

Morphologies of grafted membranes were shown in Fig. 8. For both linear and crosslinked PNIPAm-grafted membranes, the pores near the top surface of membrane were apparently filled with grafted PNIPAm. With the presence of crosslinker, even denser grafted PNIPAm were observed. It should be noted that under $800 \mathrm{w}$ of microwave irradiation, when irradiation time was longer than $15 \mathrm{~min}$, the membrane fracture was observed as shown in Fig. 9. This was suspected to be caused by the temperature in the microwave was higher than the service temperature of Nylon membrane. This irradiation that caused Nylon membrane fracture was thought to be the upper limit of grafting of Nylon membrane with PNIPAm. Thus, the optimum condition for microwave irradiation were found to be $800 \mathrm{~W}$ and 10 min for both the linear and crosslinked PNIPAM grafted on to Nylon porous membranes. For grafted membranes with different monomer concentration, a plausible explanation was that the higher concentration of monomer used, the denser grafting was being generated, especially on the top surface, and this prevented the NIPAM monomer in solution to diffuse into the pore of membrane as shown in Fig. 10. Therefore, the membrane was found to possess highly PNIPAm grafted on the top of surface which explain the increase of PNIPAm signal in FTIR and not in grafting yield that calculated by weight.

(a)

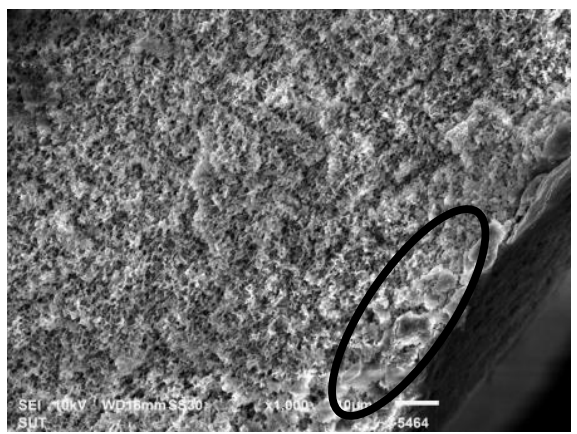

(b)

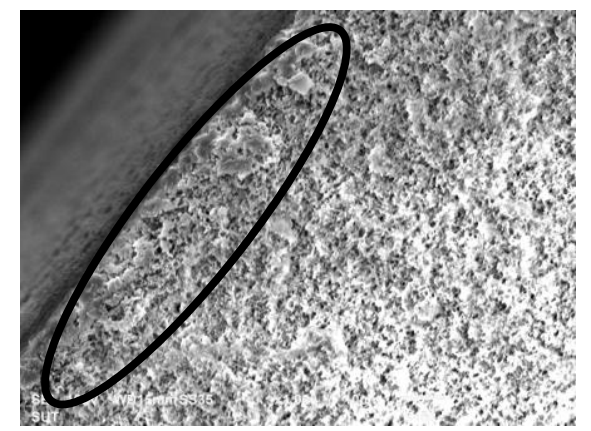

Fig. 7 SEM micrographs of cross sections of grafted samples: (a) without pressure assisted, and (b) with pressure assisted. 


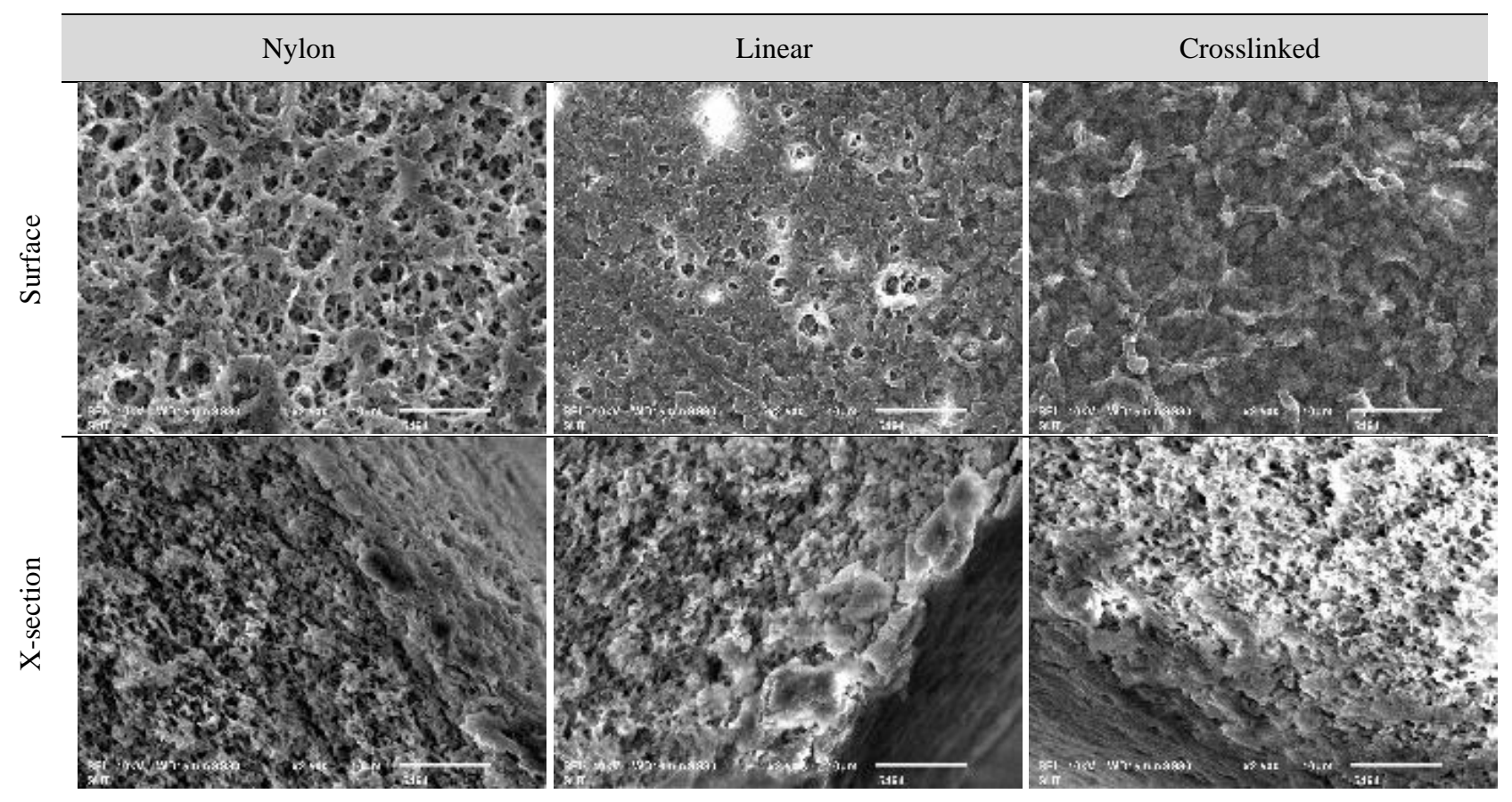

Fig. 8 SEM micrographs of surface and cross sections of samples.

(a)

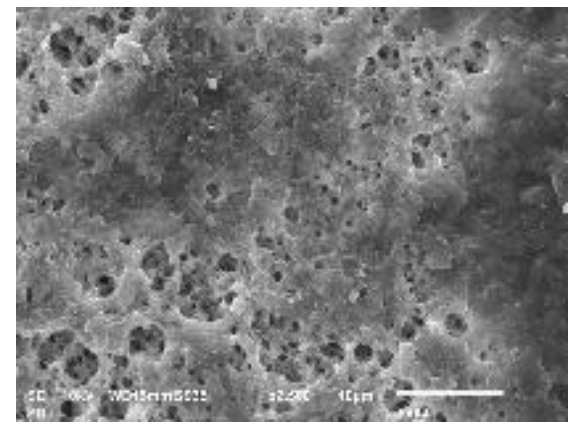

(b)

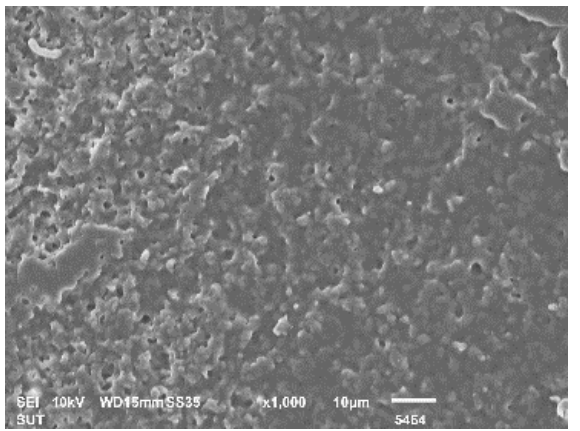

Fig. 9 SEM micrographs of a fractured microstructure of PNIPAm grafted membranes (surface) (a) $800 \mathrm{~W} 30$ min (linear) and (b) $800 \mathrm{~W} 15$ min (crosslinked) 
(a)

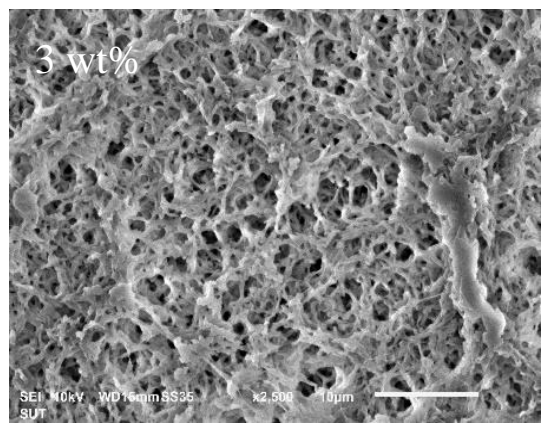

(c)

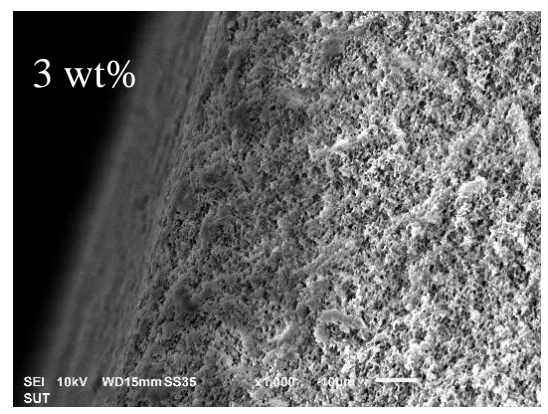

(b)

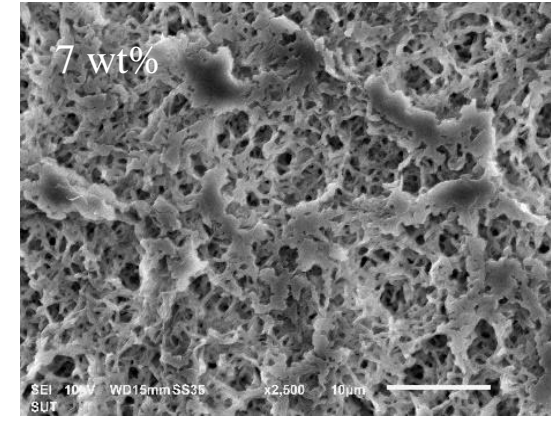

(d)

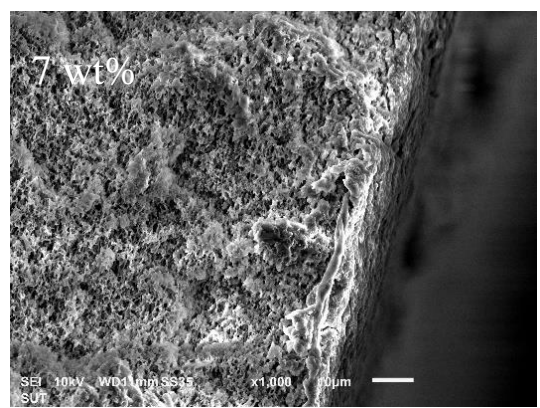

Fig. 10 SEM micrographs of samples: surface of grafted membrane with (a) $3 \mathrm{wt} \%$, (b) $7 \mathrm{wt} \%$, and cross-section of grafted membrane with (c) $3 \mathrm{wt} \%$, and (d) $7 \mathrm{wt} \%$.

\section{Conclusions}

Nylon membranes have been successfully modified by plasma peroxide method together with grafting of PNIPAm via microwave-assisted irradiation. The grafted PNIPAm chains located mainly on the top surface and at the pore entrance of the membranes. The coverage of grafted PNIPAm chains on the membrane were inhomogeneously distributed but the uniformity was improved after the use of a pressure-assisted permeation technique. Addition of the crosslinker yielded a denser grafted membrane as compared to that of without one. The grafted membranes were fractured when grafting under the condition with the irradiation time over 15 min under $800 \mathrm{w}$ because the solution temperature was higher than the operating temperature of membrane. The fractured membranes were unsuitable to be used for gating application because of unstable performances. Therefore, the PNIPAm-grafted membranes should be fabricated at a proper irradiation time of $10 \mathrm{~min}$ and under a proper output power of $800 \mathrm{w}$ to obtain the maximum grafting yield. The critical concentration of NIPAm to give highest yield was found to be at $3 \mathrm{wt} \%$. Membranes with different structures (linear chains or crosslinked networks) and different grafting yield are expected to affect their gating performance. The gating performance and thermo-responsive properties of the grafted membranes are under an ongoing investigation and going to be reported in the near future.

\section{References}

Acciaro, R., Gilányi, T. and Varga, I., Preparation of monodisperse poly( $N$-isopropylacrylamide) microgel particles with homogenous cross-link density distribution, Langmuir, Vol.27, No.12 (2011), pp.7917-7925.

Chen, Y.-C., Xie, R., Yang, M., Li, P.-F., Zhu, X.-L. and Chu, L.-Y., Gating characteristics of thermo-responsive membranes with grafted linear and crosslinked poly( $N$-isopropylacrylamide) gates, Chemical Engineering \& Technology, Vol.32, No.4 (2009), pp.622-631.

Choi, Y.-J., Yamaguchi, T. and Nakao, S.-i., A novel separation system using porous thermosensitive membranes, Industrial \& Engineering Chemistry Research, Vol.39, No.7 (2000), pp.2491-2495.

Chu Liangyin, Z.J., Chen, W., Niitsuma, T., Yamaguchi, T. and Nakao, S.-i., Effect of graft yield on the thermo-responsive permeability through porous membranes with plasma-grafted poly ( $N$-isopropylacrylamide) gates, Chin.J.Chem.Eng., Vol.11, No.3 (2003), pp.269-275.

Chung-Yang, C., Trong-Ming, D. and Wen-Yen, C., Synthesis and properties of chitosan-based thermo- and pHresponsive nanoparticles and application in drug release, Journal of Polymer Science Part A: Polymer Chemistry, 
Vol.47, No.11 (2009), pp.2798-2810.

Liu, J., Bai, S., Jin, Q., Li, C. and Yang, Q., Enhanced thermostability of enzymes accommodated in thermo-responsive nanopores, Chemical Science, Vol.3, No.12 (2012), pp.3398-3402.

Murray, M., Charlesworth, D., Swires, L., Riby, P., Cook, J., Chowdhry, B. Z. and Snowden, M. J., Microwave synthesis of the colloidal poly( $N$-isopropylacrylamide) microgel system, Journal of the Chemical Society, Faraday Transactions, Vol.90, No.13 (1994), pp.1999-2000.

Ohashi, H., Chi, X., Kuroki, H. and Yamaguchi, T., Response sensitivity of a gating membrane related to grafted polymer characteristics, Industrial \& Engineering Chemistry Research, Vol.55, No.6 (2016), pp.1575-1581.

Oliver, K. C., Controlled microwave heating in modern organic synthesis, Angewandte Chemie International Edition, Vol.43, No.46 (2004), pp.6250-6284.

Schild, H. G., Poly( $N$-isopropylacrylamide): experiment, theory and application, Progress in Polymer Science, Vol.17, No.2 (1992), pp.163-249.

Stile, R. A., Healy, K. E., Thermo-responsive peptide-modified hydrogels for tissue regeneration, Biomacromolecules Vol.2, No.1 (2001), pp.185-94.

Trongsatitkul, T. and Budhlall, B., Multicore-shell PNIPAm-co-PEGMa microcapsules for cell encapsulation, Langmuir, Vol.27, No.22 (2011), pp.13468-13480.

Xiaoling, W. and Marian, G. M., Grafting of poly( $N$-isopropylacrylamide) onto nylon and polystyrene surfaces by atmospheric plasma treatment followed with free radical graft copolymerization, Journal of Applied Polymer Science, Vol.104, No.6 (2007), pp.3614-3621.

Xie, R., Chu, L.-Y., Chen, W.-M., Xiao, W., Wang, H.-D. and Qu, J.-B., Characterization of microstructure of poly(Nisopropylacrylamide)-grafted polycarbonate track-etched membranes prepared by plasma-graft pore-filling polymerization, Journal of Membrane Science, Vol.258, No.1 (2005), pp.157-166.

Xie, R., Li, Y. and Chu, L.-Y, Preparation of thermo-responsive gating membranes with controllable response temperature, Journal of Membrane Science, Vol.289, No.1 (2007), pp.76-85.

Xu, F. J., Zhao, J. P., Kang, E. T., Neoh, K. G. and Li, J., Functionalization of nylon membranes via surface-initiated atom-transfer radical polymerization, Langmuir, Vol.23, No.16 (2007), pp.8585-8592.

Xueqin, C., Hidenori, O. and Takeo, Y., Plasma-induced graft polymerization inside pores of porous substrates assisted by an infiltration agent in acidic conditions, Plasma Processes and Polymers, Vol.11, No.4 (2014), pp.306-314.

Yamaguchi, T., Ito, T., Sato, T., Shinbo, T. and Nakao, S.-i., Development of a fast response molecular recognition ion gating membrane, Journal of the American Chemical Society, Vol.121, No.16 (1999), pp.4078-4079.

Yang, M., Chu, L. Y., Li, Y., Zhao, X. J., Song, H. and Chen, W. M., Thermo-responsive gating characteristics of poly( $N$ isopropylacrylamide)-grafted membranes, Chemical Engineering \& Technology, Vol.29, No.5 (2006), pp.631-636. 